\title{
Anderson Pereira
}

\section{Otimização baseada em confiabilidade: aplicação a treliças espaciais}

\section{TESE DE DOUTORADO}

DEPARTAMENTO DE ENGENHARIA CIVIL

Programa de Pós-graduação em Engenharia Civil 
Anderson Pereira

\section{Otimização baseada em confiabilidade: aplicação a treliças espaciais}

Tese apresentada como requisito parcial para obtenção do título de Doutor pelo Programa de Pós-graduação em Engenharia Civil da PUC-Rio. Área de Concentração: Estruturas

Orientador : Prof. Luiz Fernando Martha Co-Orientador: Prof. Luiz Eloy Vaz 
Anderson Pereira

\title{
Otimização baseada em confiabilidade: aplicação a treliças espaciais
}

Tese apresentada como requisito parcial para obtenção do título de Doutor pelo Programa de Pós-graduação em Engenharia Civil da PUC-Rio. Aprovada pela Comissão Examinadora abaixo assinada.

Prof. Luiz Fernando Martha

Orientador Departamento de Engenharia Civil - PUC-Rio

Prof. Luiz Eloy Vaz Co-Orientador UFRJ

\author{
Prof. Paulo Batista Gonçalves \\ PUC-Rio
}

Prof. Raul Rosas e Silva

PUC-Rio

Prof. Luís Volnei Sudati Sagrilo

COPPE/UFRJ

Prof. José Herskovits Norman

COPPE/UFRJ

Prof. José Eugênio Leal

Coordenador Setorial do Centro Técnico Científico - PUC-Rio 
Todos os direitos reservados. É proibida a reprodução total ou parcial do trabalho sem autorização da universidade, do autor e do orientador.

\section{Anderson Pereira}

Graduou-se em Engenharia Civil na UDESC/Joinville (Universidade do Estado de Santa Catarina) em 2000. Fez curso de mestrado em Estruturas no Programa de Pós-Graduação em Engenharia Civil da PUC-Rio pelo qual recebeu o título de mestre no ano de 2002. Interesses acadêmicos em áreas de pesquisa que envolvam otimização de estruturas, programação matemática, análise não-linear, análise de confiabilidade e elementos finitos.

Ficha Catalográfica

Pereira, Anderson

Otimização baseada em confiabilidade: aplicação a treliças espaciais / Anderson Pereira; orientador: Luiz Fernando Martha; co-orientador: Luiz Eloy Vaz. - Rio de Janeiro : PUC-Rio, Departamento de Engenharia Civil, 2007.

v., 145 f: il. ; $29,7 \mathrm{~cm}$

1. Tese (doutorado) - Pontifícia Universidade Católica do Rio de Janeiro, Departamento de Engenharia Civil.

Inclui referências bibliográficas.

1. Engenharia Civil - Tese. 2. Otimização. 3. Análise Não Linear. 4. Análise de Sensibilidade. 5. Análise de Confiabilidade. 6. Programação Matemática. 7. Otimização Baseada em Confiabilidade. I. Martha, Luiz Fernando. II. Vaz, Luiz Eloy. III. Pontifícia Universidade Católica do Rio de Janeiro. Departamento de Engenharia Civil. IV. Título. 


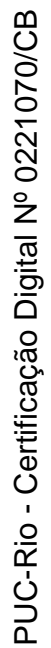

À Janaína por tudo. 


\section{Agradecimentos}

Aos meus pais, pelo apoio e por todos os gestos de carinho e otimismo.

Aos meus orientadores Luiz Eloy Vaz e Luiz Fernando Martha pelo estímulo e parceria para a realização deste trabalho.

Ao amigo Ivan, do TeCGraf (Grupo de Tecnologia em Computação Gráfica), pelo apoio, pelas excelentes discussões e sugestões ao longo deste trabalho.

Ao grande amigo Sandoval, pelas sugestões, pelo apoio e pela amizade consolidada ao longo deste trabalho.

A todos os amigos e colegas da PUC-Rio, do TeCGraf, do vôlei de praia e do chope pelos momentos de descontração, essenciais para a realização deste trabalho.

A todas as pessoas que, direta ou indiretamente, contribuíram para a realização deste trabalho. Em especial Galvão, Ataliba, Saré, Joabson, Ramires, Maurício e Pasquetti.

Aos professores do Departamento de Engenharia Civil da PUC-Rio, por todos os conhecimentos transmitidos durante a pós-graduação.

Aos funcionários da PUC-Rio e do TeCGraf, pela eficiência e amizade. Em especial à Ana Roxo, Rita, e Claudinei.

À minha esposa Janaína, pelo carinho, compreensão, paciência e constante apoio que fizeram possível a realização deste trabalho.

Ao CNPq, à PUC-Rio e ao TeCGraf, pelos auxílios concedidos. 


\section{Resumo}

Pereira, Anderson; Martha, Luiz Fernando; Vaz, Luiz Eloy. Otimização baseada em confiabilidade: aplicação a treliças espaciais. Rio de Janeiro, 2007. 145p. Tese de Doutorado - Departamento de Engenharia Civil, Pontifícia Universidade Católica do Rio de Janeiro.

No projeto de estruturas de engenharia há, freqüentemente, incertezas associadas às propriedades dos materiais, às propriedades geométricas e aos carregamentos. A maneira mais comum e tradicional para se levar em conta estas incertezas é através da definição dos valores de projeto como o resultado do produto do valor característico das variáveis aleatórias por um fator parcial de segurança. Esta solução, no entanto, falha ao não permitir a quantificação da confiabilidade do projeto ótimo uma vez que um fator grande de segurança pode não significar uma confiabilidade mais alta. Para se considerar a natureza probabilística de quantidades como propriedades dos materiais, carregamentos, etc., tem-se que identificar e definir estas quantidades como variáveis aleatórias no modelo de análise. Desta maneira, a probabilidade de falha (ou a confiabilidade) de uma estrutura sujeita a uma restrição de desempenho na forma de uma função de estado limite pode, então, ser calculada e formulada como uma restrição num problema de otimização. Neste trabalho, restrições probabilísticas são incorporadas ao esquema tradicional de otimização estrutural. A formulação e os métodos numéricos para este processo, comumente chamado de otimização baseada em confiabilidade, são descritos. O objetivo principal é apresentar um sistema computacional capaz de resolver problemas de otimização de forma e de dimensões de treliças espaciais baseado em confiabilidade. Podem ser consideradas como variáveis, determinísticas ou aleatórias, as seções transversais, as coordenadas nodais, as propriedades dos materiais (módulo de elasticidade e tensão de escoamento) e os carregamentos. De maneira a tratar os problemas de instabilidade global são considerados os efeitos da não-linearidade geométrica no comportamento da estrutura e uma restrição formulada para uma função de estado limite associada à carga de colapso é incluída. Funções de estado limite referentes aos deslocamentos e às tensões também são consideradas. A flambagem global das barras é considerada por meio da carga crítica de Euler.

\section{Palavras-chave}

Otimização. Análise Não Linear. Análise de Sensibilidade. Análise de Confiabilidade. Programação Matemática. Otimização Baseada em Confiabilidade. 


\section{Abstract}

Pereira, Anderson; Martha, Luiz Fernando; Vaz, Luiz Eloy. Reliability based optimization: application to space trusses. Rio de Janeiro, 2007. 145p. PhD Thesis - Department of Engenharia Civil, Pontifícia Universidade Católica do Rio de Janeiro.

Uncertainties associated with random variables, such as, the material proprieties and loads, are inherent to the design of structures. These uncertainties are traditionally taken into account in the project before the design by defining design values for the random variables. The design values of the random variables are obtained from statistical properties of the random variables and from partial safety factors. Once these values are defined the variables are treated as deterministic variables in the design process. This approach has been followed in the conventional design optimization and in many design codes such as the Brazilian code for the design of steel and concrete structures. This simple approach, however, does not allow an estimate of the structural reliability of the resulting project which may have a low (unsafe structure) or a very high (expensive structure) reliability. To overcome this problem a reliability analysis must be incorporated into the traditional design optimization. Design optimization, incorporating reliability analyses, has been denoted Reliability-Based Design Optimization (RBDO). In RBDO, the constraints are defined in terms of the probabilities of failure associated with some prescribed failure functions and therefore, it requires, as in the reliability analysis, the definition of the random variables and information about their statistical properties. In this work, RBDO is applied to the shape and sizing optimization of spatial trusses considering geometric nonlinearities. The constraints considered in the RBDO problem are related to the following failure mechanisms: to the global collapse (limit load), to local buckling and yield stress and to serviceability conditions (displacement bounds). The algorithms used for solving the optimization problem and for performing the reliability analysis are described.

\section{Keywords}

Optimization. Nonlinear Analysis. Sensitivity Analysis. Reliability Analysis. Mathematical Programming. Reliability Based Design Optimization. 


\section{Sumário}

\begin{tabular}{ll}
\hline Lista de Símbolos, Siglas e Abreviaturas & 14
\end{tabular}

$\begin{array}{lll}1 & \text { Introdução } & 20\end{array}$

1.1 Considerações Iniciais e Objetivos 20

\begin{tabular}{lll}
\hline 1.2 & Escopo do Trabalho & 22
\end{tabular}

$\begin{array}{lll}1.3 & \text { Revisão Bibliográfica } & 24\end{array}$

2 Análise Não-Linear Geométrica 27

$\begin{array}{lll}2.1 & \text { Comentários Iniciais } & 27\end{array}$

2.2 Comportamento Não-Linear, Análise e Projeto 27

2.2 .1 Fontes de Não-Linearidade 28

2.3 Formulação para a Análise Não-Linear Geométrica de Estruturas Reticuladas

$\begin{array}{lll}2.3 .1 & \text { Descrição do Problema } & 29\end{array}$

\begin{tabular}{lll}
\hline 2.3 .2 & Princípio dos Deslocamentos Virtuais & 30
\end{tabular}

2.3.3 Montagem das Equações da Estrutura 32

2.4 Aplicação a Elementos de Treliça Espacial 32

2.4 .1 Medida de Deformação 33

\begin{tabular}{|lll}
2.4 .2 & Medida de Tensão & 34
\end{tabular}

\begin{tabular}{|lll}
\hline 2.4 .3 & Vetor de Forças Internas & 34
\end{tabular}

2.4.4 Matriz de Rigidez 34

2.5 Estratégias de Solução para Problemas Não-Lineares 35

2.5 .1 Análise Incremental-Iterativa 36

$\begin{array}{lll}2.5 .2 & \text { Incremento Automático de Carga } & 39\end{array}$

$\begin{array}{lll}2.5 .3 & \text { Estratégias de Iteração } & 41\end{array}$

2.5 .4 Critérios de Convergência 43

2.6 Determinação dos Pontos Críticos 44

2.7 Exemplos de Análise Estrutural 45

2.7 .1 Treliça Assimétrica em Forma de Arco 45

2.7 .2 Treliça Espacial de 24 Elementos 46

$\begin{array}{lll}3 & \text { Análise de Sensibilidade } & 50\end{array}$

$\begin{array}{lll}3.1 & \text { Considerações Gerais } & 50\end{array}$

3.2 Método Analítico 51

3.2 .1 Sensibilidade dos Deslocamentos Nodais 52

3.2.2 Sensibilidade da Carga Limite 53

$\begin{array}{lll}3.2 .3 & \text { Sensibilidade da Carga Crítica Linear } & 54\end{array}$

3.2.4 Sensibilidade das Deformações e Tensões 54

3.2 .5 Sensibilidade do Vetor de Forças Internas 55

3.2 .6 Sensibilidade do Vetor de Cargas Externas 55

3.2.7 Sensibilidade da Matriz de Rigidez Elástica 56

3.2.8 Sensibilidade da Matriz de Rigidez Geométrica 56

3.3 Método das Diferenças Finitas (MDF) 57

3.4 Exemplos de Análise de Sensibilidade 58 
\begin{tabular}{lll}
\hline 3.4 .1 & Treliça Espacial de 24 Elementos & 58
\end{tabular}

$4 \quad$ Análise de Confiabilidade Estrutural $\quad 62$

4.1 Modelagem das Incertezas 62

$\begin{array}{lll}4.1 .1 & \text { Distribuições de Probabilidades } & 63\end{array}$

\begin{tabular}{|lll}
\hline 4.1 .2 & Correlação Entre Variáveis Aleatórias & 63
\end{tabular}

4.1 .3 Função Conjunta de Probabilidades 64

$\begin{array}{llll}4.2 & \text { Função de Performance } & 65\end{array}$

4.3 Métodos de Análise de Confiabilidade 66

4.3.1 Método Valor Médio (MV) 66

4.3.2 Métodos Baseados no Ponto de Projeto 67

4.3.3 Amostragem por Importância $\quad 70$

$\begin{array}{lll}4.4 \text { Análise de Sensibilidade } & 72\end{array}$

4.5 Exemplos de Análise de Confiabilidade 73

$\begin{array}{lll}4.5 .1 & \text { Coluna com } 45 \text { barras } & 73\end{array}$

$\begin{array}{lll}5 & \text { Otimização Considerando Incertezas } & \mathbf{7 8}\end{array}$

$\begin{array}{lll}5.1 & \text { Otimização Determinística } & 78\end{array}$

$\begin{array}{lll}5.2 & \text { Otimização Baseada em Confiabilidade } & 79\end{array}$

5.3 Método de Duplo Laço para RBDO 79

$5.4 \quad$ Linearizações da função de estado limite $\quad 81$

5.5 Ponto Inicial nos Métodos de Busca pelo MPP 82

5.6 Análise de Sensibilidade em RBDO 82

$\begin{array}{lll}5.7 & \text { Exemplos } & 83\end{array}$

$\begin{array}{lll}5.7 .1 & \text { Coluna Retangular Curta } & 84\end{array}$

\begin{tabular}{lll}
\hline 5.7 .2 & Viga em Balanço & 86
\end{tabular}

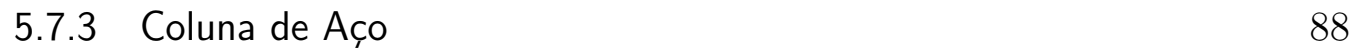

$\begin{array}{lll}5.8 & \text { Comentários Sobre os Exemplos } & 91\end{array}$

6 Otimização Baseada em Confiabilidade: Aplicação a Treliças Espaciais $\quad 93$

\begin{tabular}{lll}
\hline 6.1 & Considerações Gerais & 93
\end{tabular}

6.1.1 Consideração da Segurança nos Projetos 94

$\begin{array}{lll}6.2 \text { Metodologia de Otimização } & 96\end{array}$

6.3 Formulação do Problema de Otimização 97

$\begin{array}{lll}6.3 .1 & \text { Fatores de escala } & 98\end{array}$

6.3.2 Cálculo dos Gradientes 99

6.4 Implementação da Formulação 99

\begin{tabular}{lll}
\hline 6.5 & Exemplos & 102
\end{tabular}

$\begin{array}{lll}6.5 .1 & \text { Treliça de } 10 \text { Barras } & 102\end{array}$

\begin{tabular}{lll}
\hline 6.5 .2 & Treliça Espacial de 24 Elementos & 107
\end{tabular}

$\begin{array}{lll}7 & \text { Conclusões e Sugestões } & 112\end{array}$

\begin{tabular}{lll}
\hline 7.1 & Sugestões para Trabalhos Futuros & 114
\end{tabular}

\begin{tabular}{lr}
\hline Referências Bibliográficas & 116
\end{tabular}

\begin{tabular}{lll}
\hline A Variáveis Randômicas & 125
\end{tabular}

\begin{tabular}{|lll}
\hline A.1 Valores Característicos de uma Variável Randômica & 125
\end{tabular}

\begin{tabular}{lll}
\hline A.2 Distribuições de Probabilidades & 126
\end{tabular}

\begin{tabular}{lll}
\hline A.2.1 & Distribuição Normal ou Gaussiana & 127
\end{tabular} 
\begin{tabular}{lll}
\hline A.2.2 & Distribuição Lognormal & 127
\end{tabular}

\begin{tabular}{lll}
\hline A.2.3 & Distribuição Uniforme & 128
\end{tabular}

\begin{tabular}{lll}
\hline A.2.4 & Distribuição Gamma & 128
\end{tabular}

\begin{tabular}{lll}
\hline A.2.5 & Distribuição Beta & 129
\end{tabular}

\begin{tabular}{lll}
\hline A.2.6 & Distribuição Gumbel & 129
\end{tabular}

\begin{tabular}{lll}
\hline A.2.7 & Distribuição Tipo I Mínimos & 130
\end{tabular}

A.2.8 Distribuição Tipo II Máximos 130

\begin{tabular}{lll}
\hline A.2.9 & Distribuição Tipo III Mínimos & 131
\end{tabular}

A.2.10 Distribuição Weibull 131

B $\quad$ Algoritmos de Otimização 132

B.1 Formulação do Problema 132

\begin{tabular}{lll}
\hline B.2 & Condições de Ótimo & 133
\end{tabular}

B.3 $\quad$ Forma Geral dos Algoritmos de Otimização 134

B.4 Método de Newton para Problemas de Otimização sem Restrição 135

B.5 Busca Linear 136

B.6 Programação Quadrática 136

B.7 Algoritmo de Han-Powell - Programação Quadrática Seqüencial 137

B.7.1 Etapas do Algoritmo Não-Linear Han-Powell (SQP) 139

B.8 Método dos Pontos Interiores 140

B.8.1 Etapas do Algoritmo de Pontos Interiores (IP) 142

B.9 Algoritmos de Otimização para Análise de Confiabilidade 144

B.9.1 Algoritmo (Hasofer-Lind-Rackwitz-Fiessler) HLRF 144

B.10 Implementação 145 


\section{Lista de figuras}

2.1 Elemento de treliça. 33

2.2 Curva carga-deslocamento. 35

2.3 Solução incremental-iterativa: sistema com um grau de liberdade [25]. 38

2.4 Variação do sinal do parâmetro de rigidez generalizado (GSP). $\quad 41$

2.5 Pontos críticos de uma estrutura [58]. 44

2.6 Treliça assimétrica em forma de arco. 45

2.7 Treliça assimétrica em forma de arco - resposta estrutural do deslocamento vertical dos nós 8 e 13 . 46

2.8 Treliça espacial de 24 elementos. 48

2.9 Treliça espacial de 24 elementos - resposta estrutural para o carregamento $P_{1}$.

2.10 Treliça espacial de 24 elementos - resposta estrutural para os carregamentos $P_{1}$ e $P_{2}$.

3.1 Imprecisão da sensibilidade via diferenças finitas em problemas nãolineares [68]. 58

3.2 Treliça espacial de 24 elementos - Efeito da perturbação relativa $\eta$ \begin{tabular}{|l|l|l} 
na sensibilidade do deslocamento $q_{3}^{1}$ em relação a área $A_{1}$. & 59
\end{tabular}

3.3 Treliça espacial de 24 elementos - Sensibilidades dos deslocamentos através dos métodos analítico (DDM) e diferenças finitas. 60

3.4 Treliça espacial de 24 elementos - Deslocamento vertical do nó 1 e a sensibilidade em relação ao módulo de elasticidade.

4.1 Representação gráfica dos métodos FORM e SORM.

4.2 Representação gráfica dos métodos MCS e IS.

$\begin{array}{lll}4.3 & \text { Coluna com } 45 \text { barras. } & 74\end{array}$

4.4 Coluna com 45 barras - curvas de equilíbrio. 75

$\begin{array}{lll}5.1 & \text { Viga em balanço. } & 87\end{array}$

5.2 Coluna de aço - dependência de $\beta$ sobre o custo máximo admissível $\begin{array}{ll}\text { da estrutura [42]. } & 91\end{array}$

6.1 Situações encontradas na análise estrutural [58]. 96

6.2 RBDO-Fluxograma [41]. 100

6.3 Treliça de 10 barras. 102

6.4 Cantoneira de abas iguais - Relação entre a área da seção transversal e o momento mínimo de inércia. 103

6.5 Treliça de 10 barras - Projeto ótimo: (a) presente trabalho; (b) Stocki et. al [74].

6.6 Treliça de 10 barras - Restrições e função objetivo ao longo das iterações.

6.7 Treliça espacial de 24 elementos.

6.8 Treliça espacial de 24 elementos - Restrições e função objetivo ao longo das iterações. 
6.9 Treliça espacial de 24 elementos - Variação do volume em função do tipo de distribuição e do c.o.v. $\left[X_{27}\right]$.

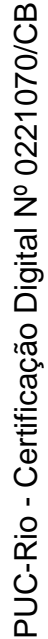




\section{Lista de tabelas}

2.1 Treliça assimétrica em forma de arco - valores de carga crítica e deslocamento vertical dos nós 8 e 13 .

2.2 Treliça espacial de 24 elementos - coordenadas nodais.

2.3 Treliça espacial de 24 elementos - valores de carga crítica e deslocamento vertical do nó 1 relativo a este nível de carregamento. 48

2.4 Treliça espacial de 24 elementos - valores de carga crítica e deslocamento vertical do nó 1 relativo a este nível de carregamento.

3.1 Treliça espacial de 24 elementos - Sensibilidades dos deslocamentos através do método analítico.

3.2 Treliça espacial de 24 elementos - Sensibilidades da carga crítica obtidas via DDM.

4.1 Distribuições de probabilidades.

4.2 Coluna com 45 barras - Seções transversais e materiais das barras.

74

4.3 Variáveis aleatórias do problema da coluna com 45 barras.

4.4 Coluna com 45 barras - Resultados com 22 variáveis para a função de falha associada ao deslocamento.

4.5 Coluna com 45 barras - Resultados com 4 variáveis para a função de falha associada ao deslocamento.

4.6 Coluna com 45 barras - Resultados para a função de falha associada à carga crítica linearizada.

5.1 RIA vs. PMA (Youn \& Choi [82]).

5.2 Não-linearidades das restrições probabilísticas (Youn \& Choi [82]).

5.3 Valores usuais dos parâmetros dos algoritmos.

5.4 Variáveis aleatórias do problema coluna retangular curta. 84

5.5 Coluna retangular curta - Resultados. 86

5.6 Coluna retangular curta - Resultados para diferentes pontos de partida.

5.7 Coluna retangular curta - Comparação dos resultados. 87

5.8 Variáveis aleatórias do problema da viga em balanço. 87

5.9 Viga em balanço - Resultados.

5.10 Viga em balanço - Resultados para diferentes pontos de partida. $\quad 89$

5.11 Viga em balanço - Comparação dos resultados. 89

$\begin{array}{ll}5.12 \text { Coluna de aço - Variáveis aleatórias. } & 89\end{array}$

5.13 Coluna de aço - resultados. 90

5.14 Coluna de aço - Comparação dos resultados. 91

6.1 Treliça com 10 barras - variáveis randômicas e de projeto. 104

6.2 Treliça espacial de 24 elementos - variáveis randômicas e de projeto. 109

6.3 Treliça espacial de 24 elementos - Resultados para diversos tipos de distribuição da variável $X_{27}$.

B.1 Divisão dos problemas de Programação Matemática. 134 


\section{Lista de Símbolos, Siglas e Abreviaturas}

\section{Caracteres latinos}

$\mathbf{a}_{i}$

A

$A_{i}$

A

$b$

$b_{0}$

$b_{i}$

$b_{z}, g_{z}$

b

$\mathbf{b}_{0}$

B

$\mathbf{B}^{0}$

$\mathbf{B}^{n l}$

$\overline{\mathbf{B}}$

$c$

$c_{i}(\cdot)$

c

C

C

d

D

$\Delta l$

$E$

$E(\cdot)$

$f(\cdot)$

f

$f_{X}$

F

$F_{X}$

$g_{i}(b, \mathbf{q}(b))$

g

$G(\mathbf{u}), g(\mathbf{x})$
Gradiente das restrição $c_{i} ; \mathbf{a}_{i}=\nabla c_{i}(\mathbf{b})$

Área da seção transversal

Área da seção transversal do elemento $i$

Matriz dos gradientes das restrições

Variável de projeto

Aproximação inicial da diagonal da Hessiana

Termos independentes das restrições

Parâmetros do algoritmo SQP

Vetor de variáveis: aleatórias ou de projeto, vetor dos coeficientes das forças de massa

Vetor inicial das variáveis de projeto

Aproximação da Hessiana, matriz que relaciona deslocamentos e deformações

Parcela constante da matriz B

Parcela linear da matriz $\mathbf{B}$

Matriz que relaciona os incrementos de deslocamento e deformações; $\overline{\mathbf{B}}=\mathbf{B}^{0}+\mathbf{B}^{n l}$

Parâmetro de penalidade

Restrição do problema de otimização

Vetor das restrições

Confiabilidade; $C=1-P_{f}$

Matriz diagonal contendo os valores das restrições, constante da equação de restrição (determinação do caminho de equilíbrio)

Direção de busca

Matriz constitutiva

Comprimento de arco da trajetória de equilíbrio

Módulo de elasticidade

Valor médio, média, ou valor esperado

Função objetivo

Vetor de forças internas do elemento

Função densidade de probabilidade (PDF); $f_{X}(x)=\frac{d F_{X}(x)}{d x}$

Vetor de forças internas

Função cumulativa de distribuição (CDF)

Restrição $i$ no problema de otimização

Gradiente da função objetivo; $\mathbf{g}=\nabla f(\cdot)$

Função de performance; a falha é caracterizada por $G(\mathbf{u})<$ $0, g(\mathbf{x})<0$

$\mathbf{h} \quad$ Vetor de variáveis de projeto; $\mathbf{h}=\left[h_{1}, h_{2}, \ldots, h_{n}\right]^{T}$

H Deslocamento generalizado 


\section{Caracteres latinos (continuação)}

$H^{(\omega+1)}, k$ Constantes da equação de restrição (determinação do caminho de equilíbrio)

$\mathbf{H} \quad$ Hessiana da função objetivo; $\mathbf{H}=\nabla^{2} f(\cdot)$

$I(\mathbf{u}) \quad$ Função indicadora; $I(\mathbf{u})=1$ se $g(\mathbf{x}) \leq 0$ e $I(\mathbf{u})=0$ nos outros casos

$\mathbf{J}_{\mathbf{u}, \mathbf{x}} \quad$ Jacobiano da transformação probabilística

$k_{a}, k_{e}, k_{f}$ Parâmetros do algoritmo de pontos interiores

$\mathbf{K}^{0} \quad$ Matriz de rigidez elástica linear padrão

$\mathbf{K}^{\sigma} \quad$ Matriz de rigidez geométrica (ou de tensões iniciais)

$\mathbf{K}^{\text {nl}} \quad$ Matriz de rigidez de deslocamentos iniciais

$L \quad$ Comprimento do elemento

$\mathcal{L}(\cdot) \quad$ Função Lagrangeana

$\mathbf{L}_{o} \quad$ Matriz triangular inferior da decomposição de Cholesky da matriz de correlação $\mathbf{R}_{o}$

$n_{r} \quad$ Número de iterações para o reinício da aproximação da Hessiana

ncc Número de casos de carregamento

$n r \quad$ Número de restrições

nvp Número de variáveis de projeto

$N_{d} \quad$ Número de iterações desejadas para cada incremento

$N_{i} \quad$ Função de forma

${ }^{t} N \quad$ Número de iterações que foram necessárias para convergir no passo de carga anterior

N Matriz das funções de forma

$p(t) \quad$ Função unidimensional utilizada na busca linear

$P \quad$ Carga concentrada

$P(\cdot) \quad$ Função de probabilidade

$P_{f} \quad$ Probabilidade de falha

$\tilde{q} \quad$ Campo de deslocamentos

q Vetor de deslocamentos nodais

$\mathbf{q}_{\mathbf{g}} \quad$ Parcela de $\delta \mathbf{q}$ referente às forças de referência $\mathbf{Q}_{r e f}$

$\mathbf{q}_{\mathbf{r}} \quad$ Parcela de $\delta \mathbf{q}$ referente às forças de residuais $\mathbf{R}$

$\mathbf{q}_{\mathbf{t}} \quad$ Vetor de deslocamentos tangenciais

Q Vetor de cargas externas

$\mathbf{Q}_{\text {ref }} \quad$ Vetor de cargas externas de referência

$r_{i} \quad$ Fatores de penalidades na busca linear

$\mathbf{R} \quad$ Vetor resíduo nodal, matriz de correlação

$\mathbf{R}_{o} \quad$ Matriz de correlação modificada

$\operatorname{sign}(\cdot) \quad$ Sinal da função: \pm 1

$t \quad$ Tamanho do passo a ser dado ao longo da direção de busca

$t_{i} \quad$ Força de tração atuando na superfície $\Gamma_{t}$

$t_{1} l_{1} \quad$ Tolerância para convergência do algoritmo SQP

$\mathrm{tol}_{2}$ Tolerância para violação de restrições do algoritmo SQP

$t_{3}$ Tolerância para convergência do algoritmo IP

$\mathbf{T} \quad$ Transformação de $\mathbf{U}$ para $\mathbf{X}$ 


\section{Caracteres latinos (continuação)}

$u, v, w \quad$ Componentes do campo de deslocamentos

$\mathbf{u} \quad$ Variáveis aleatórias normais padrão correlacionadas; $\mathbf{u}=$ $\left[u_{1}, u_{2}, \ldots, u_{n}\right]^{T}$

$\mathbf{u}_{G(\mathbf{u})=0}^{*} \quad$ Ponto mais provável de falha (MPP) na formulação RIA.

$\mathbf{u}_{\beta=\beta_{t}}^{*} \quad$ MPP na formulação PMA.

$V \quad$ Volume

$\mathrm{d} V \quad$ Elemento diferencial de volume

$\operatorname{Var}(\mathrm{X}) \quad$ Variância de $X ; \operatorname{Var}(\mathrm{X})=E\left(X^{2}\right)-\mu_{X}^{2}$

$\mathbf{v} \quad$ Autovetor associado com o autovalor nulo da matriz $\mathbf{K}$, modo de flambagem da estrutura

$\mathbf{x} \quad$ Vetor de variáveis aleatórias; $\mathbf{x}=\left[X_{1}, X_{2}, \ldots, X_{n}\right]^{T}$

$X \quad$ Variável aleatória

$z_{1}, z_{2}, z_{3} \quad$ Coordenadas cartesianas

$z_{i}^{j} \quad$ Coordenada na direção $i$ do nó $j$

$\mathbf{z}$ Variáveis aleatórias normais padrão não correlacionadas; $\mathbf{z}=$ $\left[z_{1}, z_{2}, \ldots, z_{n}\right]^{T}$

$\mathbf{W}^{*} \quad$ Hessiana da função Lagrangeana; $\mathbf{W}^{*}=\nabla^{2} \mathcal{L}\left(\mathbf{b}^{*}\right)$

$Y \quad$ Variável aleatória auxiliar; $Y=\frac{X-\mu}{\sigma}$

\section{Caracteres gregos}

$\alpha \quad$ Seqüencia de valores utilizados na busca linear

$\alpha_{i} \quad$ Cosseno diretor com relação a variável $u_{i}$ do vetor normal a superfície de falha no ponto de projeto e no espaço das variáveis reduzidas

$\beta \quad$ Índice de confiabilidade; $\beta=-\Phi^{-1}\left(P_{f}\right)$

$\beta_{t} \quad$ Índice de confiabilidade alvo

$\delta(\cdot) \quad$ Correção iterativa, indicador variacional

$\Delta(\cdot) \quad$ Perturbação finita, incremento

$\delta_{X} \quad$ Coeficiente de variação de $X ; \delta_{X}=$ c.o.v. $=\frac{\sigma_{x}}{\mu_{x}}$

$\boldsymbol{\epsilon} \quad$ Vetor das deformações

$\epsilon_{i j} \quad$ Deformações de Green-Lagrange

$\boldsymbol{\eta}$ Parâmetros determinísticos

$\gamma \quad$ Parâmetro de controle na busca linear

$\Gamma(x)$ Função Gamma

$\Gamma_{t} \quad$ Superfície

$\lambda \quad$ Fator de carga, parâmetro da distribuição Lognormal

$\boldsymbol{\Lambda} \quad$ Matriz diagonal para a qual $\boldsymbol{\Lambda}_{i i}=\kappa_{i}$

$\boldsymbol{\mu} \quad$ Média dos parâmetros randômicos $\mathbf{x} ; \boldsymbol{\mu}=\left[\mu_{1}, \mu_{2}, \ldots, \mu_{n}\right]^{T}$

$\kappa \quad$ Multiplicadores de Lagrange

$\mu_{X} \quad$ Média da variável aleatória $X$

$\nabla(\cdot) \quad$ Operador gradiente

$\Phi(\cdot) \quad$ Função de distribuição cumulativa normal padrão

$\rho$ Coeficiente de deflexão da direção de busca, massa específica, coeficiente de correlação 


\section{Caracteres gregos (continuação)}

$\boldsymbol{\sigma} \quad$ Vetor das tensões

$\boldsymbol{\theta}$ Parâmetros das variáveis randômicas (média e desvio padrão)

$\varphi \quad$ Função densidade de probabilidade normal padrão

$\varphi_{2} \quad$ Função densidade de probabilidade bidimensional normal padrão

$\xi \quad$ Parâmetro da distribuição Lognormal

$\zeta_{1} \quad$ Fator de convergência baseado em relações de força

$\zeta_{2} \quad$ Fator de convergência baseado em relações de deslocamentos

$\zeta \quad$ Tolerância ao resíduo requerida no processo de convergência

$\sigma_{X} \quad$ Desvio padrão de $X ; \sigma_{X}=\sqrt{\operatorname{Var}(\mathrm{X})}$

$\rho_{o} \quad$ Coeficiente de correlação aproximado

\section{Sobrescritos e Subscritos}

${ }^{t}(\cdot) \quad$ Função avaliada no instante $t$

${ }^{t+\Delta t}(\cdot) \quad$ Função avaliada no instante $t+\Delta t$

$(\cdot)^{(\omega)} \quad$ Função avaliada no iteração $\omega$

$(\cdot)^{*} \quad$ Função avaliada no ponto crítico ou no ponto ótimo

$(\cdot)^{T} \quad$ Transposta do vetor ou matriz

$(\cdot)_{i j} \quad$ Notação indicial

$(\cdot)^{l} \quad$ Limite inferior da variável de projeto

$(\cdot)^{u} \quad$ Limite superior da variável de projeto

$(\cdot)^{-1},[\cdot]^{-1}$ Inversa de uma matriz

\section{Siglas e Abreviaturas}

AMV Método Valor Médio Avançado (do inglês Advanced Mean Value methods)

ASM Método Adjunto de Sensibilidade (do inglês Adjoint System Method)

CDF Função Cumulativa de Distribuição (do inglês Cumulative Distribution Function)

DDM Método da Diferenciação Direta (do inglês Direct Differentiation Method)

DDO Otimização Determinística (do inglês Deterministic Design Optimization)

DEC Departamento de Engenharia Civil

DEM Departamento de Engenharia Mecânica

DLM Método de Duplo Laço (do inglês Double-loop RBDO methods) 


\section{Siglas e Abreviaturas (continuação)}

DSA Análise de Sensibilidade (do inglês Design Sensitivity Analysis)

FEA Análise de Elementos Finitos (do inglês Finite element Analysis)

FORM Método de Confiabilidade de Primeira Ordem (do inglês First Order Reliability Method)

GDCM Método do Controle de Deslocamento Generalizado (do inglês Generalized Displacement Control Method)

GL Deformações de Green-Lagrange

GSP Parâmetro de Rigidez Geral (do inglês General Stiffness Parameter)

HLRF Algoritmo de Hasofer-Lind-Rackwitz-Fiessler

IP Algoritmo de Pontos Interiores (do inglês Interior Point)

IRA Problema de Confiabilidade Inverso (do inglês Inverse Reliability Analysis)

IS Amostragem por Pontos de Importância (do inglês Importance Sampling)

KKT Karush-Kuhn-Tucker Problema Linear Complementar

MCS Simulação de Monte Carlo (do inglês Monte Carlo Simulation)

MDF Método das Diferenças Finitas

MPP Ponto Mais Provável de Falha (do inglês Most Probable Point)

MVFOSM Método Valor Médio Primeira Ordem Segundo Momento (do inglês Mean-Value-First-Order Second-Moment)

MV Método Valor Médio (do inglês Mean Value method, ou $M V$ FOSM)

NLPQLP Algoritmo de Programação Quadrática Seqüencial

OOP Programação Orientada a Objetos (do inglês Object Oriented Programming)

OPT Módulo de Otimização

PBDO Otimização Baseada em Possibilidade (do inglês PossibilityBased Design Optimization)

PDF Função Densidade de Probabilidade (do inglês Probability Density Function)

PK2 Tensões de Piola-Kirchhoff II

PMA Formulação de Medida de Performance (do inglês Performance Measure Approach)

PM Programação Matemática

PQ Programação Quadrática

PSF Fator de Suficiência Probabilística (do inglês Probability Sufficiency Factor) 


\section{Siglas e Abreviaturas (continuação)}

RBDO Otimização Baseada em Confiabilidade (do inglês Reliability Based Design Optimization)

RIA Formulação do Índice de Confiabilidade (do inglês Reliability Index Approach)

RLA Referencial Lagrangeano Atualizado

RLT Referencial Lagrangeano Total

RSA Aproximação por Superfície de Resposta (do inglês Response Surface Approximation)

SORM Método de Confiabilidade de Segunda Ordem (do inglês Second Order Reliability Method)

SQP Programação Quadrática Seqüencial (do inglês Sequencial Quadratic Programming)

c.o.v Coeficiente de Variação 